\title{
Advanced trauma life support courses
}

\author{
Teaching thoroughness and efficiency under pressure
}

Trauma causes about 22000 deaths each year in Britain. ${ }^{1}$ The commonest cause of death in the first four decades of life, it has important economic implications, both through the cost of treatment and the loss of productivity.

In 1988 a working party of the Royal College of Surgeons criticised the standard of trauma care and recommended that doctors managing trauma should undertake advanced trauma life support courses. ${ }^{2}$ These are educational programmes designed to teach medical staff a systematic approach to managing seriously injured patients. At a time when priorities may get confused they ensure that diagnoses are made while resuscitative life saving measures continue.

The courses originated in the United States after several studies showed that American trauma services were inadequate. Specialised trauma services were regarded as the solution, ${ }^{34}$ but it was recognised that these would not raise the standard of care given in places remote from such services. In 1978 the Lincoln Medical Education Foundation and the South East Nebraska Emergency Medical Services developed a prototype advanced trauma life support course with the aim of improving trauma care in community hospitals. A year later, after several revisions, the American College of Surgeons' committee on trauma adopted the present advanced trauma life support course..$^{5}$ Such has been the impact and success of these courses that many American hospitals now require medical staff working in their emergency departments to be certified in advanced trauma life support as a condition of employment. ${ }^{6}$

Currently courses fall into two categories: provider and instructor. Their goal is to enable a doctor to assess and manage patients with major trauma during the "golden hour," defined as the time after injury during which lives may be saved if the appropriate resuscitation is started. ${ }^{7}$ Although based on the assumption that the severely injured patient is being managed by a singlehanded doctor, the protocols may be easily adapted to the team approach in hospital. A single member of the trauma team familiar with the system of advanced trauma life support can direct the actions of the other members of the team and improve management.

At present the content of the provider courses is the same as that used in the United States, following the manual produced by the American College of Surgeons' committee on trauma. ${ }^{5}$ To qualify candidates must apply the principles exactly as set out in the lectures and the manual.

Provider courses (for 16 candidates at a time) run for two and a half days. The approach applies the logical sequence of $\mathrm{A}=$ airway, $\mathrm{B}=$ breathing, and $\mathrm{C}=$ circulation for resuscitation and expands on it so that the injured patient is meticulously examined and resuscitated at the same time. Practical skill stations using training aids are run to familiarise doctors with the management of airways and minor surgical procedures. In the United States courses commonly use anaesthetised large animals for teaching venous cutdown, the insertion of chest drains, diagnostic peritoneal lavage, and cricothyroidotomy. British legislation prohibits this - instead, animal or human cadaveric models and video tapes of American practice are used. $^{8}$

Assessment includes multiple choice questionnaires and "moulage" (human models with simulated injuries) to test the candidates' performance under stress. A suitably made up person acts as the victim with trauma, the circumstances are described, and for 30 minutes the instructor assesses the candidate's ability to examine and resuscitate the victim ? according to the protocols of advanced trauma life support. $\overrightarrow{\vec{F}}$ Without warning, the instructor may introduce new problems, such as a tension pneumothorax that recurs or a chest drain that suddenly drains stomach contents. Assistants may $\overline{\bar{m}}$ stress and distract the candidates. Certification lasts four $\mathbb{\otimes}$ years, after which the candidate must repeat the examina- on tion.

Participants who perform well on the provider course may be invited to take part in a two and a half or three day course $\vec{\omega}$ for instructors, which supplements the core content of the provider course with additional sessions on teaching tech- 3 niques and arranging skill stations. The American College of $\omega_{0}$ Surgeons' committee on trauma has produced a set of slides covering all the core content material, thus standardising the lectures.

The first instructor course outside North America was held of in November 1988 under the auspices of the Royal College of Surgeons of England, with instructors trained in the United States. Currently the college runs three instructor courses a year along the American lines. More certified instructors should result in more provider courses; already the number of $\frac{\widehat{\partial}}{\supset}$ provider courses nationwide has increased from seven in 1989 to 46 last year. Currently there are 133 certified instructors in advanced trauma life support in the United Kingdom, and over 1000 candidates have successfully completed provider courses (A Ezekial, personal communication).

British doctors deal with less trauma than their American colleagues, ${ }^{9}$ but this is no reason to accept lower standards of $\frac{\mathbb{Q}}{\mathbb{Q}}$ trauma care. Courses in advanced trauma life support provide $\vec{F}$ an opportunity to improve the basic standards of care.

Details of forthcoming advanced trauma life support provider courses are advertised in the $B M F$ or are available from A Ezckiel, Hunterian Institute, Royal College of Surgeons of England, 35-43 Lincoln's Inn Fields, London WC2A 3PN. Typical fees, excluding accommodation, are $£ 300$ for the provider course and $£ 405$ for the instructor course.

Department of Anaesthesia,

JERRY P NOLAN Senior Registrar

Royal United Hospital,

Bath BA1 3NG

Sir Humphrey Davy Department of Anaesthesia

Bristol Royal Infirmary,

Bristol BS2 8HW

Frenchay Hospital,

Bristol BS16 1LE

(Correspondence to Dr Nolan)

1 World Health Organisation. World health statistics annual. Geneva: WHO, 1989.

2 Commission on the Provision of Surgical Services. Report of the working party on the patients with major injuries. London: Royal College of Surgeons of England, 1988.

West J, Trunkey DD, Lim RC. Systems of trauma care. Arch Surg 1979;114:455-60.

DD, Lim RC. Systens of trauma care. Arch Surg 1979 ;114: system. Ann Emerg Med 1984;13:1-10. Committee on Trauma, American College of Surgeons. Advanced trauma life support program, instructor manual. Chicago: American College of Surgeons, 1989.

6 Myers RAM. Advanced.trauma life support courses. $\mathcal{F}$ R Soc Med 1990;83:281-2.

Cowley RA, Dunham CM. Shock trauma/critical care manual. Baltimore: University Park Press, 1982:xi-v.

Eaton BD, Messent DO, Haywood IR. Animal cadaveric models for advanced life support training Ann R Coll Surg Engl 1990;72:135-9.

9 Grande CM, Nolan JP. Trauma: anaesthesia and resuscitation. In: Atkinson RS, Adams AP, eds. Recent advances in anaesthesia and analgesia. Number seventeen. Edinburgh: Churchill Livingstone, 1992:25-47. 